\title{
OUT-OF-PLANE DYNAMIC RESPONSE OF A TUFF MASONRY WALL: SHAKING TABLE TESTING AND NUMERICAL SIMULATION
}

\author{
Daniela Addessi, Enrico Cappelli, Cristina Gatta and Fabrizio Vestroni \\ Department of Structural and Geotechnical Engineering \\ Sapienza University of Rome \\ Via Eudossiana, 18, 00184, Rome, Italy \\ e-mail: \{daniela.addessi,cristina.gatta,vestroni\}@uniroma1.it
}

Keywords: Masonry, Shaking table, Out-of-plane response, Damage, Finite Element.

\begin{abstract}
The out-of-plane dynamic response of a masonry element is investigated, both experimentally and numerically. The results of shaking table tests on a tuff masonry wall, subjected to harmonic acceleration histories, are presented. An isotropic nonlocal damage-plastic model, accounting for the masonry strength-stiffness degrading and hysteresis mechanisms, is introduced in a finite element procedure to numerically describe the masonry structural response. A simplified scheme is analysed, where the wall is completely restrained at the base and free at the top. The measured top displacement history is compared with that numerically evaluated, obtaining a satisfactory agreement. Moreover, the effects of the onset and evolution of the degrading mechanisms in the masonry wall are highlighted.
\end{abstract}




\section{INTRODUCTION}

In the last decades, many efforts have been devoted to develop efficient procedures for the assessment of the seismic safety of masonry structures. This is a challenging task, especially related to the wide spread of masonry buildings in the Italian historical and architectural heritage. Moreover, the recent Italian earthquakes have further highlighted the relevance of this issue. The analysis of the earthquake's effects on masonry structures [1, 2] has led to define a collapse hierarchy, considering three main mechanisms: crumbling, out- and in-plane failure. When the masonry crumbling is prevented, it has been widely assessed that the out-of-plane collapse mechanisms are the most frequent. Regarding the out-of-plane behavior of unreinforced masonry walls, several theoretical and experimental studies have highlighted that these walls show a deformable behavior during the first stage of the response. As the loading process proceeds, depending on the wall boundary conditions, the concentration of the damage in small areas of the walls leads to the formation of nonlinear hinges and the panels rotate as rigid blocks [3]. Although the out-of-plane response is affected by many factors, such as the level of axial force and the boundary conditions [4, 5], simplified structural schemes are commonly adopted. Different modeling approaches can be used [6], that is microscopic, macroscopic, multi-scale, as well as simplified macro-element formulations. All account for the masonry nonlinear constitutive behavior, by introducing damage-plastic models to describe the degrading of strength and stiffness and the hysteresis mechanisms, typically occurring in masonry response.

This study focuses on the out-of-plane dynamic response of a tuff masonry wall, subjected to harmonic acceleration histories, both experimentally and numerically. The main aim is to investigate the effects of the nonlinear phenomena, such as the onset and propagation of microcracking, on the wall dynamic response. Experimental tests are performed on a one-direction horizontal shaking table, considering a simple scheme for the wall, rigidly connected to the table at the base and free at the top. Sinusoidal acceleration motions with increasing amplitudes are assigned. Such simple inputs are selected, instead of natural earthquake histories, to better characterize the wall dynamic response and its dependence on the main properties of the loading history, such as frequency and amplitude [7]. The wall is equipped with various instruments, to measure the displacement and acceleration response, as well as the variation of the natural frequencies after each loading stage. A numerical study of the analyzed wall is also performed, by adopting the macroscopic damage-plastic model for masonry presented in [7, 8] and limiting the analysis to the range where the wall exhibits a deformable behavior, before the transition to the rigid block response. The adoption of a macroscopic modeling approach represents a fair compromise between accuracy of results and computational effort. The model mechanical parameters are identified on the basis of experimental tests and a finite element (FE) procedure is adopted to perform the numerical analysis. The experimental and numerical outcomes are compared in terms of top displacement response and damage distribution, giving a satisfactory agreement regarding the main features of the wall nonlinear dynamic behavior.

\section{DAMAGE-PLASTIC MODEL}

A macroscopic isotropic damage-plastic model is adopted to investigate the dynamic response of a tuff masonry wall. This model, formulated on the basis of that presented in [8], is able to capture:

- the degrading processes developing in masonry material for prevailing tensile and compressive states, through the introduction of two different scalar damage variables $D_{t}$ and $D_{c}$, respectively; 
- the onset and growth of irreversible strains by means of a classical J2 plasticity model with kinematic hardening;

- the unilateral effect linked to the re-closure of the tensile cracks, when the material undergoes compression strain states.

Thus, the following stress-strain relationship is proposed:

$$
\boldsymbol{\sigma}=\left[\left(1-D_{t}\right) \alpha_{t}+\left(1-D_{c}\right) \alpha_{c}\right]^{2} \mathbf{C}\left(\varepsilon-\varepsilon^{p}\right)
$$

where $\mathbf{C}$ is the elastic constitutive matrix for plane-stress conditions, and $\varepsilon$ and $\varepsilon^{p}$ denote the total and plastic strain vectors, respectively. $D_{t}$ and $D_{c}$ are the damage scalar variables for prevailing tensile and compression strain states, respectively. These range between 0 (initial undamaged material) and 1 (completely damaged material), and satisfy the irreversible thermodynamic constraint, that is $\dot{D}_{t / c} \geq 0$. The evolution of the damage variables is driven by two equivalent strain measures, $Y_{t}$ and $Y_{c}$, defined on the basis of the total principal strains $\hat{\varepsilon}_{i}$, as:

$$
Y_{t}=\sqrt{\sum_{i=1}^{3}\left\langle e_{i}\right\rangle_{+}^{2}} \quad Y_{c}=\sqrt{\sum_{i=1}^{3}\left\langle e_{i}\right\rangle_{-}^{2}+\kappa \sum_{i=1}^{3} \sum_{j \neq i}\left\langle e_{i}\right\rangle_{-}\left\langle e_{j}\right\rangle_{-}}
$$

with,

$$
e_{i}=(1-\nu) \hat{\varepsilon}_{i}+v \sum_{j=1}^{3} \hat{\varepsilon}_{j}
$$

The weigthing coefficients $\alpha_{t}$ and $\alpha_{c}$ in Eq. (1) are defined as:

$$
\alpha_{t}=\frac{Y_{t}^{e} / Y_{t 0}}{Y_{t}^{e} / Y_{t 0}+Y_{c}^{e} / Y_{c 0}} \quad \alpha_{c}=1-\alpha_{t},
$$

where the principal elastic strains $\hat{\varepsilon}_{i}^{e}$ are introduced to evaluate $Y_{t / c}^{e}$. Note that the parameter $\alpha_{t}$ takes the unit value for pure tensile states and vanishes in pure compression. Therefore, in all possible strain states, $\alpha_{t / c}$ varies in the range $[0,1]$.

Two different damage limit functions, $F_{t}$ and $F_{c}$, are defined to rule the evolution of $D_{t / c}$, as follows:

$$
\begin{aligned}
& F_{t}=\left(Y_{t}-Y_{t 0}\right)-D_{t}\left(a_{t} Y_{t}+b_{t}\right) \\
& F_{c}=\left(Y_{c}-Y_{c 0}\right)-D_{c}\left(a_{c} Y_{c}+b_{c}\right)
\end{aligned}
$$

Mac'Auley brackets $\langle\bullet\rangle_{+/-}$in Eq. (2) select the positive and negative part of the quantity; $Y_{t 0}$ and $Y_{c 0}$ are the damage initial thresholds in tension and compression; $a_{t / c}$ and $b_{t / c}$ govern the shape of the constitutive law, affecting mainly the slope of softening branches and the peak strengths, respectively. Note that the equivalent compressive strain $Y_{c}$ in Eq. (2) contains the material parameter $\kappa$, which influences the shape of the limit function for bi-axial compression states.

To overcome the mesh-dependency problems emerging in presence of strain-softening behavior, a classical nonlocal integral procedure is adopted [9], and the integral definition, $\tilde{Y}_{t / c}$, of the damage associated variables is introduced. 
The classical J2 plasticity model for plane stress problems [10] is adopted to account for the onset and growth of the irreversible strains, denoting with $\sigma_{y}$ and $H_{k}$ the yield stress and the kinematic hardening, respectively.

In Figure 1, the uni-axial cyclic stress-strain law is depicted with reference to the mechanical parameters in Table 1. Note that the adopted constitutive law correctly reproduces the unilateral effect, as well as the hysteresis loops. The presented formulation has been introduced in a 9-node quadrilateral finite element and implemented in the FEAP code [11].

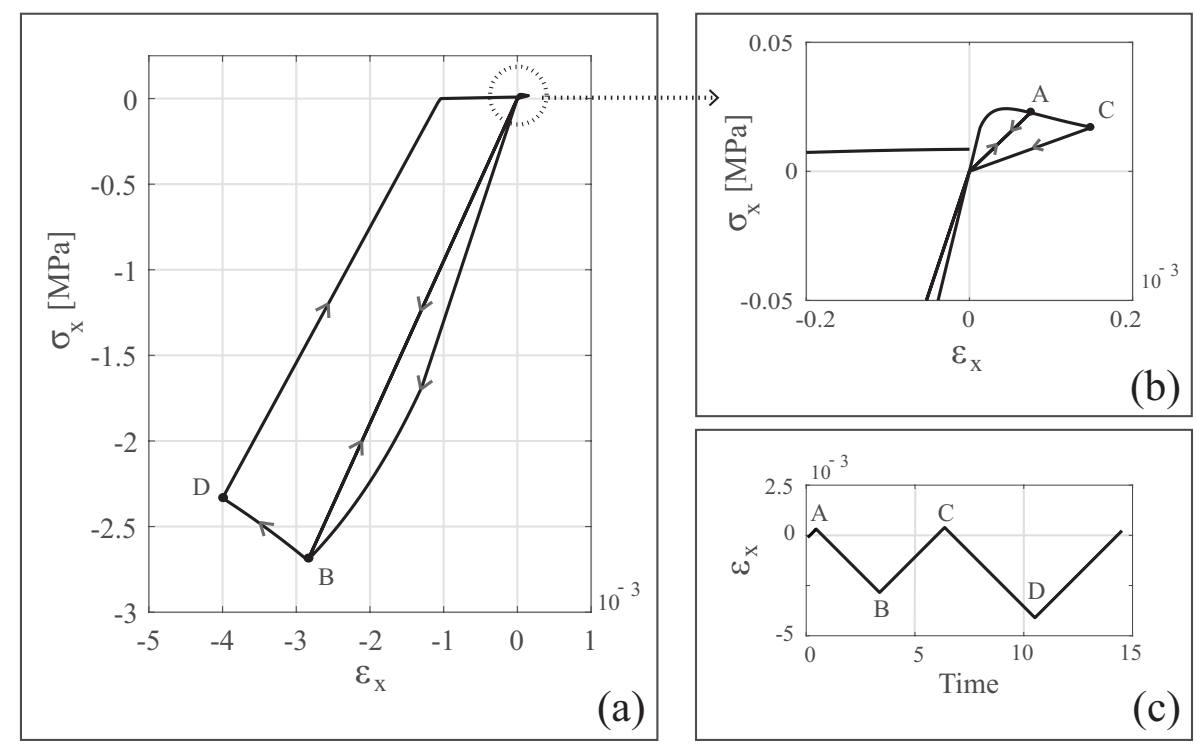

Figure 1: (a) Uni-axial cyclic stress-strain law, (b) tensile constitutive response and (c) imposed strain.

\section{EXPERIMENTAL TEST}

\subsection{Geometry and material properties}

The test specimen consists in a single leaf wall schematically shown in Figure 2(a). This is made of 19 corses of tuff bricks arranged in running bond with natural hydraulic lime mortar joints. The sizes are $H=2280 \mathrm{~mm}, L=570 \mathrm{~mm}$ and $B=260 \mathrm{~mm}$. The brick first row is clamped in a steel beam $C 300$ with the interposition of a mortar bed joint, linking the wall to the beam. Thus, the wall is considered as completely restrained at the base.

Dynamic identification tests have been performed to measure the specimen natural frequencies and the initial undamaged elastic modulus. To this end, an instrumented hammer and an accelerometer have been placed at the last corse of bricks in the middle of the wall. Figure 3 (a) shows the Fast Fourier Transform (FFT) of each impact (solid grey lines) and the average curve (black line). The results obtained by the different impacts are comparable in terms of natural frequencies. Moreover, the ratio between the first natural frequency, equal to $7.3 \mathrm{~Hz}$, and the second, equal to $42.9 \mathrm{~Hz}$, confirms that the fully restrained base condition is correctly reproduced, as this approaches the ratio analytical value equal to 5.94 , evaluated by means of the Timoshenko beam theory. The mass density of the masonry material has been measured through a load cell, resulting equal to $\rho=1577 \mathrm{~kg} / \mathrm{m}^{3}$. On the basis of the natural frequencies and the mass density values, the Young's modulus of the homogenized masonry material has been computed. 
Some other relevant material properties have been derived from previous experimental tests on the same masonry material [12].

Table 1 contains the adopted material parameters, used to perform the numerical analysis. Finally, in Figure 2(b) the numerically computed uni-axial compressive stress-strain law is compared with the experimental one, showing a good match.
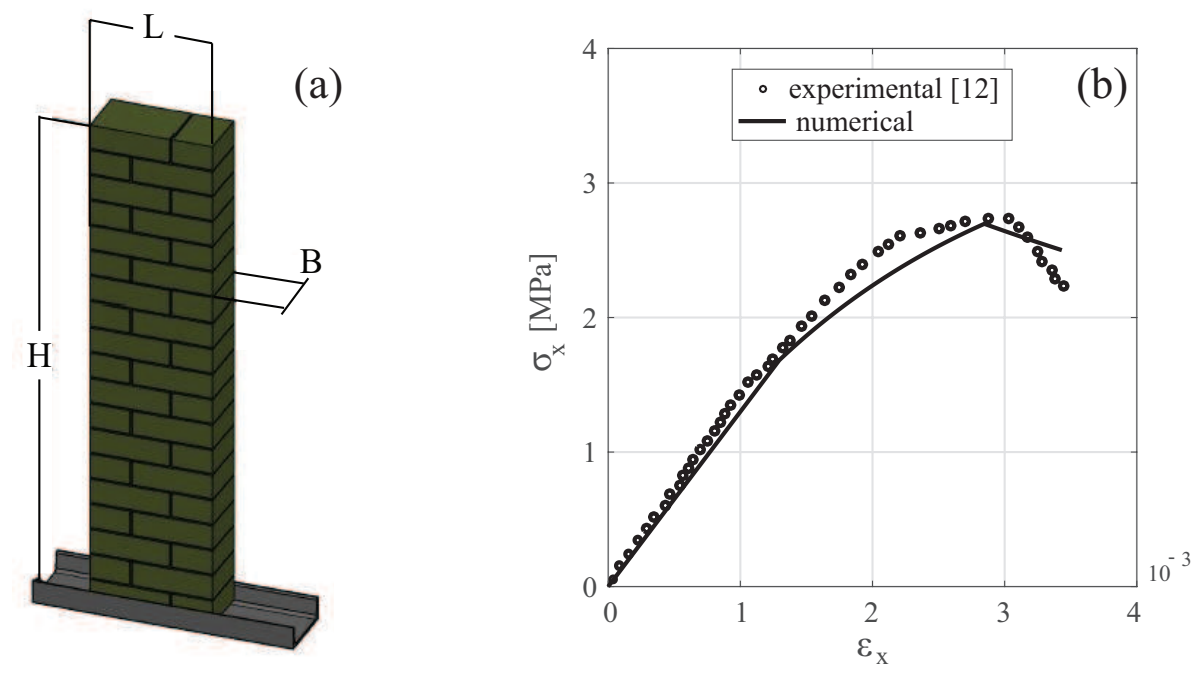

Figure 2: (a) Schematic of the specimen; (b) stress-strain curves under uni-axial compression.

\begin{tabular}{|c|c|c|c|c|c|}
\cline { 2 - 5 } \multicolumn{1}{c|}{} & Elastic parameters & \multicolumn{2}{c|}{ Plastic parameters } & \multicolumn{1}{c|}{} \\
\cline { 2 - 5 } \multicolumn{1}{c|}{} & $E[\mathrm{MPa}]$ & $\nu$ & $\sigma_{y}[\mathrm{MPa}]$ & $H_{k}[\mathrm{MPa}]$ & \multicolumn{1}{c|}{ Damage parameters } \\
1300 & 0.18 & 3.7 & $0.1 E$ & \\
\hline$Y_{t 0}$ & $Y_{c 0}$ & $a_{t}$ & $a_{c}$ & $b_{t}$ & $b_{c}$ \\
$1.0 \mathrm{E}-05$ & $1.0 \mathrm{E}-03$ & 0.995 & 0.995 & $3.5 \mathrm{E}-05$ & $6.0 \mathrm{E}-03$ \\
\hline
\end{tabular}

Table 1: Masonry material parameters.

\subsection{Test setup and input motions}

The wall in Figure 2 has been experimentally tested in the laboratory of the Department of Structural and Geotechnical Engineering of Sapienza University of Rome, placing it on a onedirection horizontal shaking table. The sizes in plane of the table are of $1500 \mathrm{~mm} \times 1500 \mathrm{~mm}$, the upper and lower bounds of the imposed displacement are $\pm 200 \mathrm{~mm}$ and the exciting frequencies can range between $[1 \div 20] \mathrm{Hz}$. The out-of-plane dynamic response of the wall, connected to the shaking table, has been investigated by measuring accelerations and displacements in the direction of motion. More in details, three linear variable displacement trasducers (LVDTs) have been placed: two at the top of one vertical side of the wall, so as to observe any torsional motions, and one at the base of the shaking table. Moreover, five accelerometers have been set: one at the base of the wall, two on the top, and the others at the center of the two 
vertical sides orthogonal to the motion direction. In Figure 4 (a) the picture and (b) the scheme of the test setup are shown: here the LVDTs and the accelerometers are depicted with square and circular markers, respectively.

Concerning the input motions, sinusoidal accelerations with different amplitudes and frequencies have been considered. Hereafter, the most relevant results are presented, corresponding to the excitation frequency $\Omega$ lower than the first natural frequency of the wall $\omega_{1}$, as the resonance frequency is moved leftward, when the degrading processes occur. Table 2 contains the amplitudes of the applied accelerations, considering $\eta=\Omega / \omega_{1}=0.65$, corresponding to the input frequency $\Omega=4.7 \mathrm{~Hz}$.
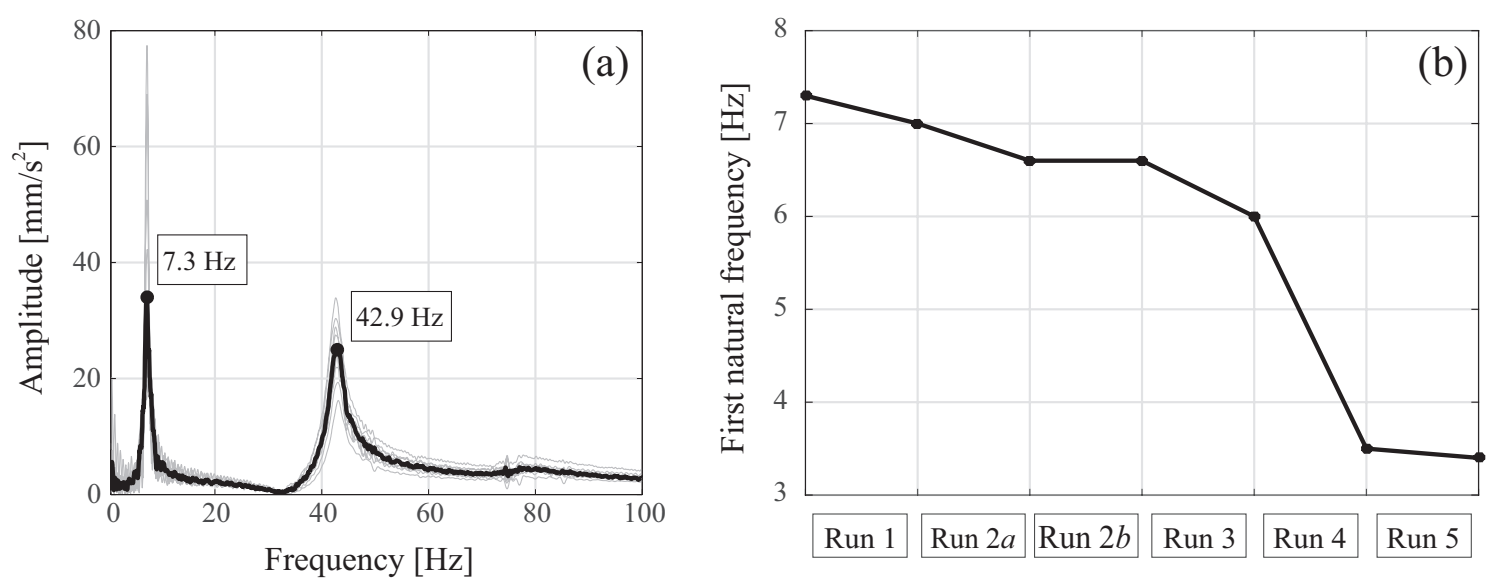

Figure 3: Natural frequencies of (a) the elastic and (b) damaged wall.

As shown in Table 2, the first applied input motions (Run 1 and 2a) immediately achieve their designed amplitude, while the others are characterized by five incoming and outgoing fading cycles. These have permitted to avoid amplified transient responses that could have caused undesirable damages, making the monitored results unsuitable.

\begin{tabular}{ccccccc}
\hline Run & 1 & $2 a$ & $2 b$ & 3 & 4 & 5 \\
Amplitude $\left[\mathrm{mm} / \mathrm{s}^{2}\right]$ & 200 & 300 & 300 & 500 & 700 & 900 \\
Fade in cycle & & & $\checkmark$ & $\checkmark$ & $\checkmark$ & $\checkmark$ \\
\hline
\end{tabular}

Table 2: List of the input sinusoidal waves.

\subsection{Experimental and numerical results}

The experimental results, in terms of time histories of the wall top relative displacement, are shown in Figure 6(a) with black line. These highlight that the wall structural response, arranged in sequence according to the imposed input motions, is strongly affected by the nonlinear degrading mechanisms evolving in the masonry. Indeed, the specimen has exhibited a degradation of the mechanical properties during the tests, causing a relevant modification of its structural response. This is also evident in Figure 3(b), where the frequencies of the damaged wall are shown. These have been deduced by the FFTs of the measured top displacement responses. The reduction of the wall first frequency points out that a progressive damage has occurred. As 


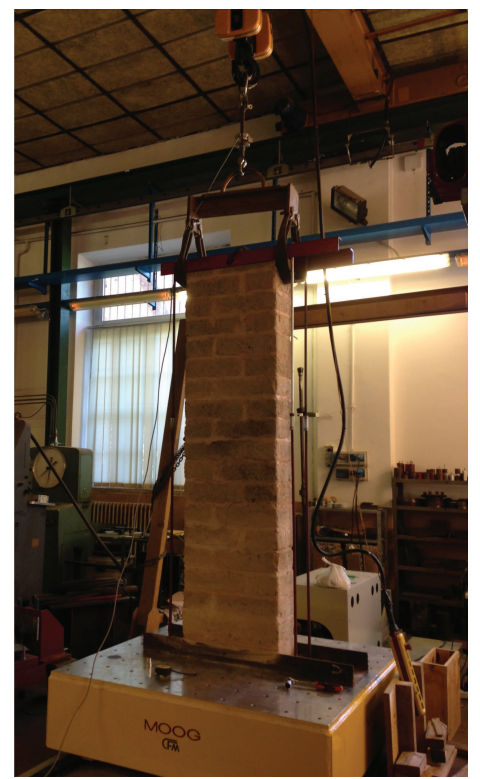

(a)

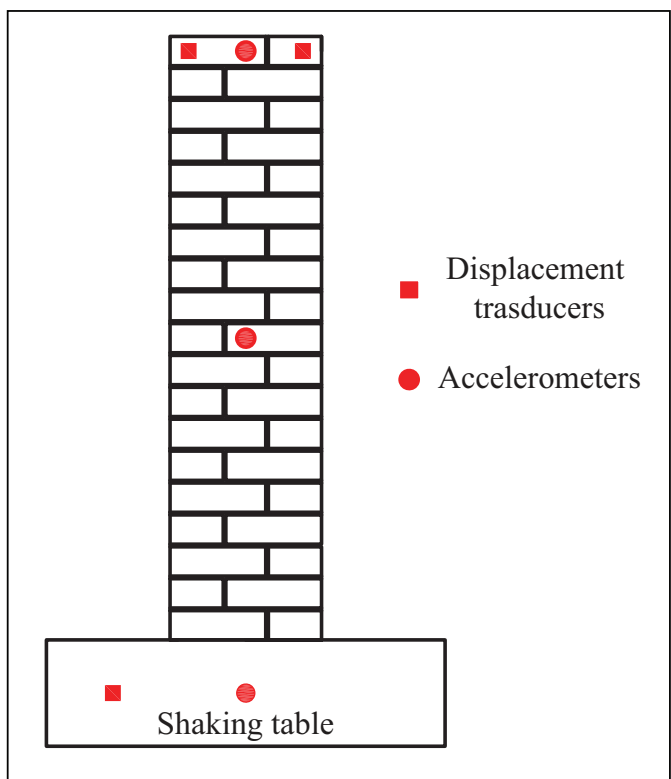

(b)

Figure 4: (a) Picture and (b) schematic of the test setup.

the amplitude of the applied acceleration has been increased, the degrading process has evolved and the first natural frequency of the damaged structure has approached to the input frequency, moving the structural element towards the resonance condition. During Run 3, the wall top displacement shows a progressive slight amplification, while during Run 4 a steep growth, typical of the resonant conditions, occurs. After this, a fairly stable response is noted, showing that the further variation of the natural frequency during Run 4 distances the structure from the resonance conditions. Furthermore, no significant plastic phenomena occur, as, due to the geometry of the wall, the damaging flexural mechanisms located at the wall base are predominant. Indeed, all the damage seems to be concentrated in a mortar bed joint at the base, which causes MODE I collapse of masonry. Concerning the numerical simulations, a mesh of $26 \times 39$-node

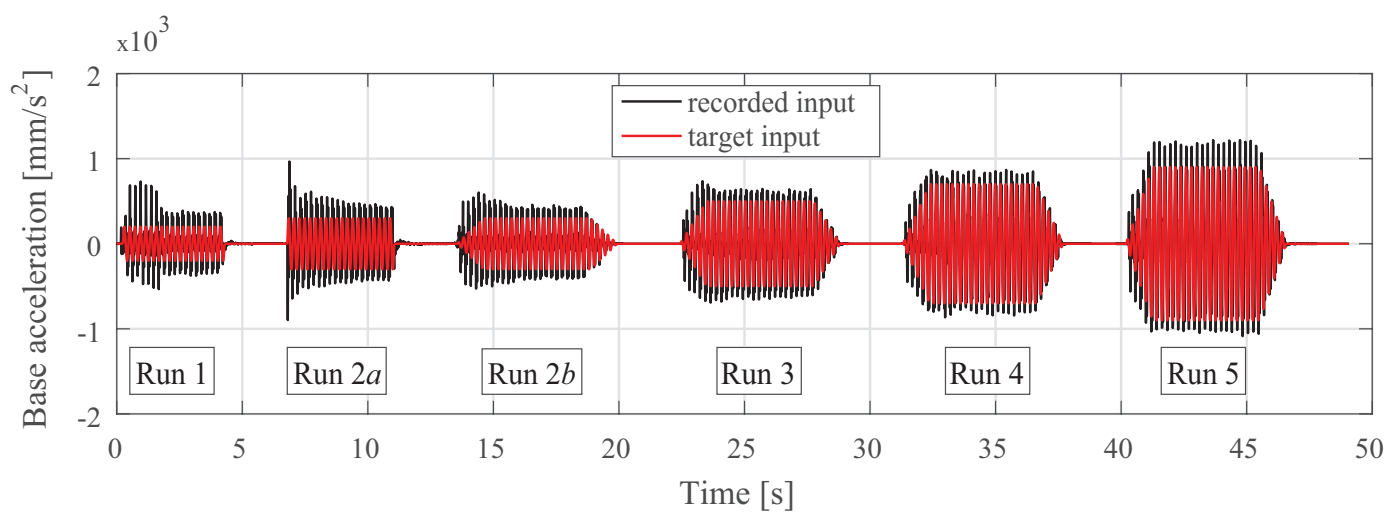

Figure 5: Comparison between the recorded and target input signals. 
quadrilateral FEs is used, setting the nonlocal radius $l_{c}=130 \mathrm{~mm}$. A damping ratio of $3 \%$ is introduced. The Newmark implicit time integration scheme is adopted to solve the FE governing equations, together with the Newton-Raphson procedure to determine the nonlinear solution in each time step. It is worth noting that a little discrepancy (see Figure 5) between the sinusoidal
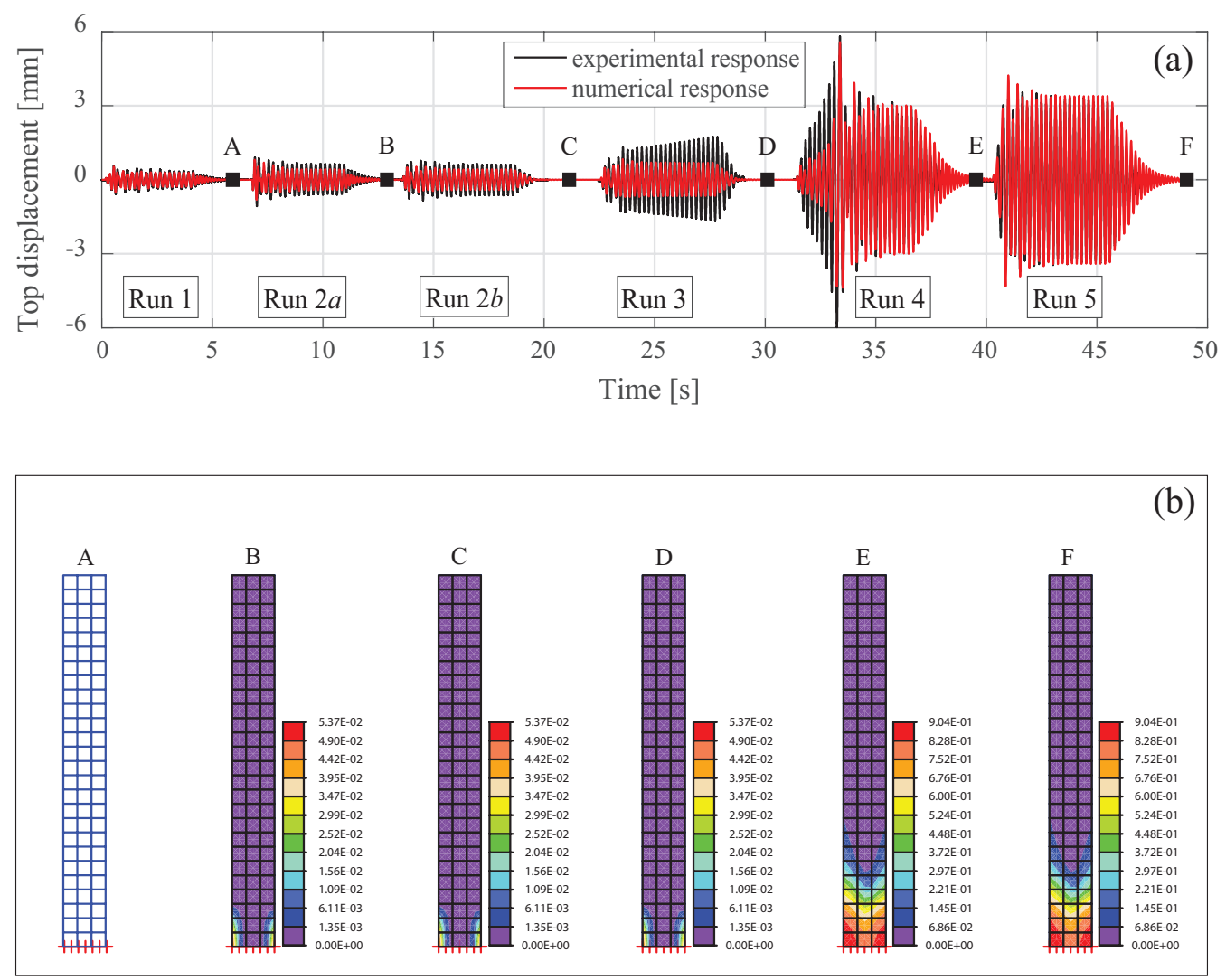

Figure 6: (a) Experimental and numerical top displacement response and (b) tensile damage distribution.

target inputs (red line) and those recorded by the shaking table (black line) has emerged, such differences resulting more evident in the case of the lower amplitude inputs. The input signals recorded by the table have been used for the numerical simulations. However, the analyses have shown that the wall overall behavior is not affected by these slight discrepancies. In Figure 6(a) the numerically obtained results are depicted in red, considering the material parameters in Table 1. The results are presented by arranging the numerical response to each input motion according to the sequence shown in Table 2, aiming at reproducing the actual pattern of the degrading process. On the overall, the model is able to describe the main aspects of the wall dynamic response. Indeed, the actual resonance condition and the maximum displacement experienced by the wall are satisfactorily matched. However, the main discrepancy between the experimental and numerical response emerges in Run 3, where the degrading process in the numerical simulation evolves much more slowly than in the experimental response. Indeed, the resonant response starts during Run 3 and ends in Run 4 in the experimental outcomes, whereas this is concentrated in Run 4 as concerns the numerical results. This is probably due to the effect of the fatigue damage, which is not included in the presented model. Furthermore, both numerical and experimental curves show slight differences in the response amplitude between 
Run 4 and 5, although the amplitude of the input signal exhibits an increase of $30 \%$. This means that the wall has departed from the resonance conditions.

In Figure 6(b), the damage maps are shown at the end of each Run. These are in agreement with the degrading processes observed during the experimental tests. During Run 1, no damage occurs in the numerical simulation, despite a decay of $4 \%$ in the first natural frequency has been experimentally estimated. However, the displacement responses are overlapped, which means that damaging process has not substantially affected the structural behavior. No increase of damage appears between Run $2 a, 2 b$ and 3 , as the maximum displacements exhibited by the wall are the same. During Run 4, where the wall experiences the resonance conditions, the formation of severe damaged zones located at bottom corners of the walls appears.

To highlight the model ability in reproducing the actual progression of the degrading process in the wall, a further numerical simulation has been performed, assigning the input sequence made of Run 3 and 4 on the initial undamaged wall. A modified degraded value of the Young's modulus, $E=1080 \mathrm{MPa}$, is adopted, set consistently with the FFT results reported in Figure 3(b). The obtained numerical response (green line) is shown in Figure 7(a), compared with the experimental curve (black line). In this case, the model better describes the experimental outcomes with respect to the results shown in Figure 6(a). Despite the numerical resonance condition is slightly shifted with respect to the actual one, there is a better match in terms of top displacement amplitude. This is also confirmed in Figure 7(b) and (c), where the FFT of the top displacement response is presented both for the experimental case (black line) and for the numerical simulations reported in Figure 6(a) (red line) and 7(a) (green line), respectively, pointing out a satisfactory agreement in terms of frequency content and amplitude. The responses are characterized by two main frequencies, that are the forcing and the natural one, and show, once again, the variation of the wall natural frequencies, when the degrading process evolves.
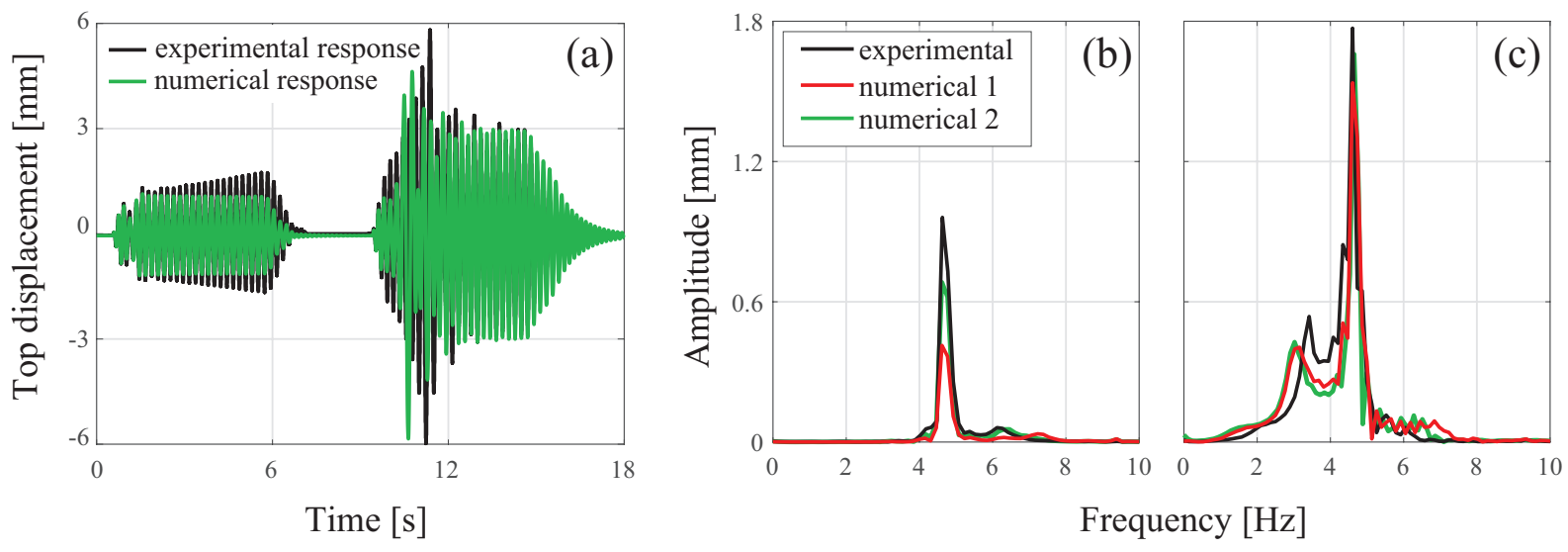

Figure 7: (a) Experimental and numerical top displacement response and their Fourier spectra during (b) Run 3 and (c) Run 4.

\section{CONCLUSIONS}

- A shaking table test has been carried out to investigate the out-of-plane dynamic behavior of a tuff masonry wall. The top displacement response of the specimen has been monitored under sinusoidal acceleration input with a fixed frequency and increasing amplitude. 
- The experimental results have highlighted the significant influence of the nonlinear mechanisms evolving in the masonry on the structural response. A reduction of the wall first frequency is associated with the onset of damage. Thus, the panel initially approaches to the resonance conditions, then, comes out when further degradation occurs.

- A finite element procedure has been implemented, introducing a nonlocal damage-plasticity model. In spite of the complexity of the phenomena, the experimental and numerical results are in good agreement, as both the resonant response and the maximum displacement experienced by the wall are satisfactorily matched. Slight differences arise regarding how the damage evolves, more regular in the experimental tests compared with numerical simulations. This could be probably due to some limitations of the adopted model, which currently does not account for the degrading process related to the repeated cycle loading.

\section{REFERENCES}

[1] V. Gattulli, E. Antonacci, F. Vestroni, Field observations and failure analysis of the Basilica S. Maria di Collemaggio after the 2009 L'Aquila earthquake. Engineering Failure Analysis, 34, 715-734, 2013.

[2] L. Sorrentino, L. Liberatore, L.D. Decanini, D. Liberatore, The performance of churches in the 2012 Emilia earthquakes. Bulletin of Earthquake Engineering, 12, 2299-2331, 2014.

[3] K. Doherty, M.C. Griffith, N. Lam, J. Wilson, Displacement-based seismic analysis for out-of-plane bending of unreinforced masonry walls. Earthquake Engineering and Structural Dynamics, 31, 833-850, 2002.

[4] D.P. Abrams, O. AlShawa, P.B. Lourenço, L. Sorrentino, Out-of-Plane Seismic Response of Unreinforced Masonry Walls: Conceptual Discussion, Research Needs, and Modeling Issues. International Journal of Architectural Heritages, 11, 22-30, 2017.

[5] T.T. Bui, A. Limam, Out-of-plane behaviour of hollow concrete block masonry walls unstrengthened and strengthened with CFRP composite. Composites: Part B, 67, 527-542, 2014.

[6] D. Addessi, S. Marfia, E. Sacco, J. Toti, Modeling Approaches for Masonry Structures. The Open Civil Engineering Journal, 8, 288-300, 2014.

[7] D. Addessi, C. Gatta, F. Vestroni, Characterization of the Dynamic Behavior of Masonry Structural Elements with Damage. J. Kruis, Y. Tsompanakis, B.H.V. Topping eds. Proceedings of the Fifteenth International Conference on Civil, Structural and Environmental Engineering Computing, Prague, Czech Republic, September 1-4, 2015.

[8] D. Addessi, C. Gatta, F. Vestroni, Static and dynamic nonlinear response of masonry walls. Submitted.

[9] P. Pijaudier-Cabot, Z.L. Bazant, Non local damage theory. Journal of Engineering Mechanics ASCE, 118(10), 1512-1533, 1987.

[10] J.C. Simo, R.L. Taylor, A return mapping algorithm for plane stress elastoplasticity. International Journal for Numerical Methods in Engineering, 22, 649-670, 1986. 
[11] R.C. Taylor, FEAP-A finite element analysis program, Version 8.3. Department of Civil and Environmental Engineering. University of California at Berkeley, 2011.

[12] G. Marcari, M. Basili, F. Vestroni, Experimental investigation of tuff masonry panels reinforced with surface bonded basalt textile-reinforced mortar. Composites Part B, 108, 131-142, 2017. 